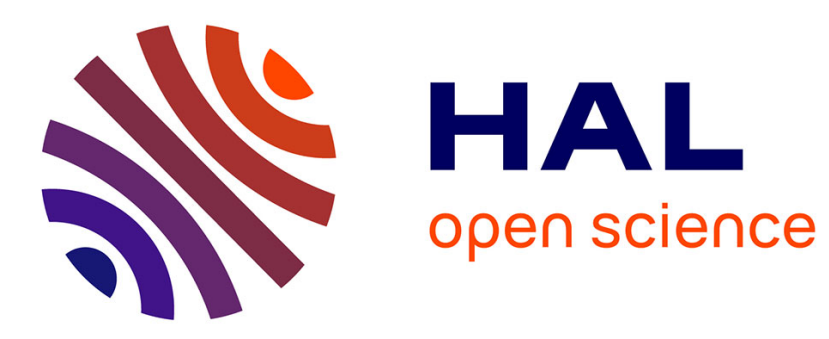

\title{
Segmentation of Retinal Arteries in Adaptive Optics Images
}

Nicolas Lermé, Florence Rossant, Isabelle Bloch, Michel Paques, Edouard Koch

\section{To cite this version:}

Nicolas Lermé, Florence Rossant, Isabelle Bloch, Michel Paques, Edouard Koch. Segmentation of Retinal Arteries in Adaptive Optics Images. 2013. hal-00868639v5

\section{HAL Id: hal-00868639 https://hal.science/hal-00868639v5}

Preprint submitted on 23 Apr 2014

HAL is a multi-disciplinary open access archive for the deposit and dissemination of scientific research documents, whether they are published or not. The documents may come from teaching and research institutions in France or abroad, or from public or private research centers.
L'archive ouverte pluridisciplinaire HAL, est destinée au dépôt et à la diffusion de documents scientifiques de niveau recherche, publiés ou non, émanant des établissements d'enseignement et de recherche français ou étrangers, des laboratoires publics ou privés. 


\title{
Segmentation of Retinal Arteries in Adaptive Optics Images
}

\author{
Nicolas Lermé* ${ }^{*}$, Florence Rossant*, Isabelle Bloch ${ }^{\dagger}$, Michel Paques ${ }^{\ddagger}$ and Edouard Koch ${ }^{\ddagger}$ \\ * LISITE, Institut Supérieur d'Électronique de Paris, Paris, France \\ † LTCI, CNRS UMR5141, Institut Mines-Télécom, Télécom ParisTech, Paris, France \\ $\ddagger$ CIC 503, Centre Hospitalier National des Quinze-Vingts, Paris, France
}

\begin{abstract}
In this paper, we present a method for automatically segmenting the walls of retinal arteries in adaptive optics images. To the best of our knowledge, this is the first method addressing this problem in such images. To achieve this goal, we propose to model these walls as four curves approximately parallel to a common reference line located near the center of vessels. Once this line is detected, the curves are simultaneously positioned as close as possible to the borders of walls using an original tracking procedure to cope with deformations along vessels. Then, their positioning is refined using a deformable model embedding a parallelism constraint. Such an approach enables us to control the distance of the curves to their reference line and improve the robustness to image noise. This model was evaluated on healthy subjects by comparing the results against segmentations from physicians. Noticeably, the error introduced by this model is smaller or very near the inter-physicians error.
\end{abstract}

Keywords: Active contours model, adaptive optics, approximate parallelism, retina imaging.

\section{INTRODUCTION}

The diseases affecting the retinal blood vessels of small diameter $(\leq 150 \mu \mathrm{m})$ such as arterial hypertension or diabetes are major causes of morbidity and mortality. According to the Public Health Agency of Canada, these diseases affected 15 to $20 \%$ of the world's adult population in 2009. Hypertensive retinopathy (HR) and diabetic retinopathy (DR) are common ocular complications of these diseases that cause modifications of vessels walls and are predictive of end-organ damage such as stroke or visual loss [1], [2]. [3] estimate that $98 \%$ of visual damages could be avoided if DR was treated in time. Accurate measurements of walls are therefore necessary to better prevent the DR and the complications of HR. However, classical fundus photographs and Doppler measurements cannot capture such level of details due to their limited spatial resolution [4].

Adaptive-Optics (AO) based cameras improve the lateral resolution of fundus photographs, thus enabling the visualization of microstructures such as photoreceptors [7], capillaries [8] and vascular walls [9], noninvasively. In the present study, the camera $\mathrm{rtx} 1$ [6] is used to acquire 2D images by flood illumination at $10 \mathrm{~Hz}$ using a $850 \mathrm{~nm}$ LED light source with a pixel-resolution of $0.8 \mu \mathrm{m}$. Flood-illumination systems usually produce noisy images making walls hardly visible. A commonly used solution is to register these images and average them to increase the signal-to-noise ratio [5]. In these images, blood vessels appear as dark elongated structures with a bright linear axial reflection, over a textured background.

Work funded by the ANR project ANR-12-TECS-0015-03 (2013-2015).

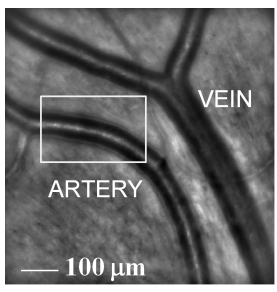

$924 \times 947$

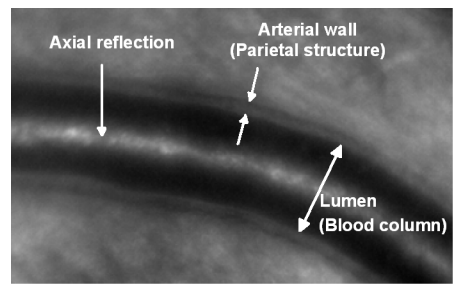

$365 \times 232$
Figure 1: A mean image produced by [5] acquired the $\mathrm{rtx} 1$ camera [6] (left) and a detailed view of an artery (right). The size of each image is indicated below it and expressed in pixels.

Outer borders of walls are however only visible along arteries and the present study will therefore focus on them. Parietal structures (arterial walls) appear as a gray line along both sides of the lumen (blood column), with a typical thickness of about $15 \%$ of the latter [10] (see Figure 1).

In this paper, we propose an automatic procedure for segmenting arterial walls in a selected region of interest 1 in the mean images produced by [5]. To the best of our knowledge, this is the first method adressing this problem in such images. This task is challenging for multiple reasons: (i) background is highly textured, (ii) lumens are globally dark but with significant intensity variation along them, (iii) axial reflections may locally show discontinuities or poor contrast, (iv) outer borders of walls are low-contrasted, (v) segments can be locally blurred due to the geometry of the retina, and (vi) deformations can occur along vessels in case of pathologies.

To overcome these difficulties, we propose a strategy exploiting geometric, radiometric and topological a priori information of vessels. More precisely, we model arterial walls as four curves approximately parallel to a common reference line located near the axial reflection. Once this line is detected, the curves are simultaneously positioned as close as possible to the borders of walls using a tracking procedure to cope with deformations along vessels. Then, their positioning is refined using an active contours approach [11] where curves evolve towards large image gradients under a parallelism constraint. Such an approach enables us to control the distance of the curves to their reference line and improve the robustness to image noise. Finally, we also want to mention that this work has recently permitted us to establish relationships between morphometric measurements and clinical parameters [12].

\footnotetext{
${ }^{1}$ In particular, we do not aim at segmenting the whole vascular tree.
} 
The rest of this paper is organized as follows. In Section II, we first summarize the steps for detecting axial reflections. Then, we detail in Section III the procedure for segmenting the arterial walls. Finally, we evaluate the relevance of the approach against manual segmentations in Section IV.

\section{AXIAL REFLECTION DETECTION}

In this section, we consider images as functions mapping points from $\Omega \subset Z^{2}$ into the interval $[0,1]$.

\section{A. Pre-processing}

The original image (see Figure 2(a)) is first enhanced by applying a median filter followed by a non-linear diffusion filter [13]. This combination of filters allows us to denoise the image and smooth the blood vessels while preserving the contrast along their edges. We denote by $I_{P}$ the resulting image (see Figure 2(b)).

\section{B. Detection of bright elongated structures}

To enhance the bright elongated structures, a white tophat transform is applied on the pre-processed image $I_{P}$ with a binary disk whose radius is about $1 / 3$ of the axial reflection diameter. We denote by $I_{T}$ the resulting image (see Figure 2(c)). Then, we binarize the image $I_{T}$ by hysteresis thresholding and denote it by $I_{E S}$ (see Figure 2(d)). Parts of the axial reflection of vessels are thus extracted, but also other bright areas of the textured background. Further processing steps are therefore necessary to discard these undesired areas.

\section{Detection of the darkest areas}

$\mathrm{K}$-means classification $(k=3)$ is performed on the preprocessed image $I_{P}$ (see Figure 2(e)) and the cluster of lowest mean intensity value provides a first binary image of the darkest regions. It is then post-processed with morphological operations to get the main connected components corresponding to the dark areas of lumens. We denote by $I_{D A}$ the resulting image (see Figure 2(f)).

\section{Extraction of vascular segments by information fusion}

A first selection of vascular segments is performed based on a simple measure of the tortuosity. Let us denote by $I_{E S}^{L}$ the binary image of a tested connected component of the image $I_{E S}$. This component is retained if

$$
\frac{\sharp I_{E S}^{L}}{\sharp\left(I_{E S}^{L} \bullet S\right)}>0.8,
$$

where $\sharp$ denotes the cardinality of a set, $\bullet$ denotes the morphological closing operator and $S$ is a binary disk whose radius is 15 pixels. Moreover, a segment of axial reflection must lie inside a dark area, and conversely, a dark region of the lumen must contain at least one axial reflection segment. We denote by $I_{E S^{\prime}}$ the binary image made of the components satisfying (1) and $I_{E S^{\prime}}^{L}$ a tested component of it. The component $I_{E S^{\prime}}^{L}$ is kept as part of an axial reflection segment if

$$
\sharp\left(I_{D A} \cap\left(I_{E S^{\prime}}^{L} \oplus S^{\prime}\right)\right)>\frac{\sharp I_{E S^{\prime}}^{L}}{5},
$$

where $\oplus$ denotes the morphological dilation operator and $S^{\prime}$ is a binary disk whose radius is 15 pixels. Notice that the radius of $S$ and $S^{\prime}$ are determined according to the minimum size of the vessels that are studied. We denote by $I_{E S^{\prime \prime}}$ the binary image made of the components satisfying (2) (see Figure 2(g)). Morphological operations are applied to $I_{D A}$, including reconstruction by dilation with the marker $I_{E S^{\prime \prime}}$, in order to get the final lumen mask $I_{L M}$ (see Figure 2(h)).

\section{E. Segment labeling and reconnection}

We first compute the skeleton of the image $I_{E S^{\prime \prime}}$ to get the end-points of the retained segments (see Section II-D). These end-points are then reconnected using minimal path techniques [14], [15]. These techniques aim at extracting curves of minimal length, in a Riemannian metric computed from the image and depending on the targeted application. A minimal path $C$ connecting two end-points $p$ and $q$ is obtained by minimizing the following functional:

$$
L[C]=\int_{p}^{q} \mathcal{P}(C(s)) d s,
$$

where $s$ denotes the curvilinear abscissa and $\mathcal{P}$ is a potential inducing the metric defined as

$\mathcal{P}(x)=w_{1}\left(1-I_{T}(x)\right)^{2}+w_{2}\left(1-I_{S M}(x)\right)^{2}+w_{3}, \quad \forall x \in \Omega$,

where $I_{S M}$ is the pruned skeleton of the lumen mask $I_{L M}$ filtered by a Gaussian of standard deviation $\sigma$ and $w_{1}, w_{2}, w_{3} \in$ $\mathbb{R}^{+}$are free parameters. These parameters are empirically set to $\sigma=10, w_{1}=0.5, w_{2}=0.45$ and $w_{3}=0.05$. In the latter expression, the first term is derived from the top-hat image $I_{T}$ (considering that the values should ideally be close to one along the axial reflection) while the second one encourages the path $C$ to pass near the middle of the lumen mask $I_{L M}$. The last term is a regularization constant. The combination of the above criteria allow for a good robustness against the variety of the encountered images.

Two end-points form a candidate pair for reconnection if they belong to the same connected component in the lumen mask $I_{L M}$ and if they do not belong to the same connected component in the image $I_{E S^{\prime \prime}}$. The candidate pairs are then processed by decreasing order of the Euclidian distance (to start with points that are close to each other) and reconnected using the above procedure. A new skeleton is then calculated, providing the axial reflection of the vessels, and the vessel branches are then labeled (see Figure 2(i)). The vessel branchs are individually regularized using a classical parametric active contour [16] with Gradient Vector Flow [17]. The lumen mask $I_{L M}$ is also labeled such that every non-null pixel receives the label of the closest branch (see Figure 2(j)).

Although the above described steps rely on a number of parameters, we empirically found that they are all stable for the images studied (including those presented in Section IV).

\section{SEGMENTATION OF ARTERIAL WALLS}

For convenience, we detail the procedure for segmenting arterial walls on a single regularized vessel branch obtained at the end of the axial reflection detection step (see Section II). We denote this regularized branch as the reference line $V(s)=$ $(x(s), y(s))^{T}$ of the vessel, parameterized by $s$. Once obtained, 


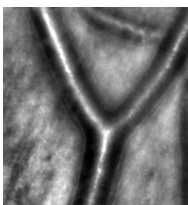

(a)

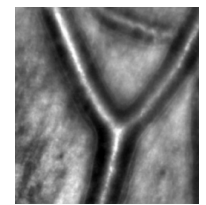

$I_{P}$ (b)

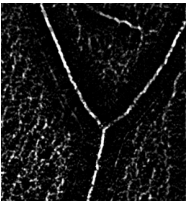

$I_{T}(\mathrm{c})$

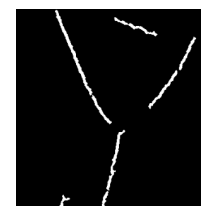

$I_{E S}$ (d)

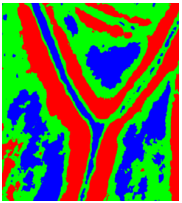

(e)

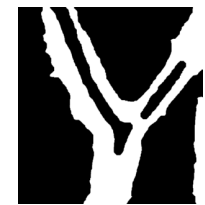

$I_{D A}$ (f)

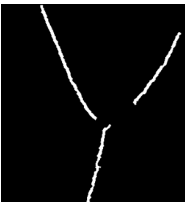

$I_{E S^{\prime \prime}}(\mathrm{g})$

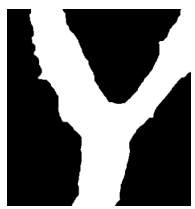

$I_{L M}(\mathrm{~h})$

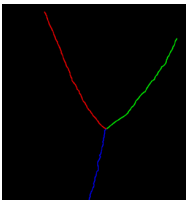

(i)

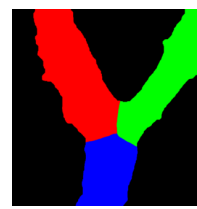

(j)

Figure 2: Steps for axial reflection detection: The source image (a) is first pre-processed (b). Dark elongated structures are detected from (b) by top-hat filtering (c) followed by hysteresis thresholding (d). Darkest areas are detected from (b) by k-means (e) followed by post-processing (f). Axial segments (g) and lumens (h) are extracted from (d) and (f). Axial segments (i) and lumens (j) are labeled from (c), (g) and the spurred skeleton of (h).

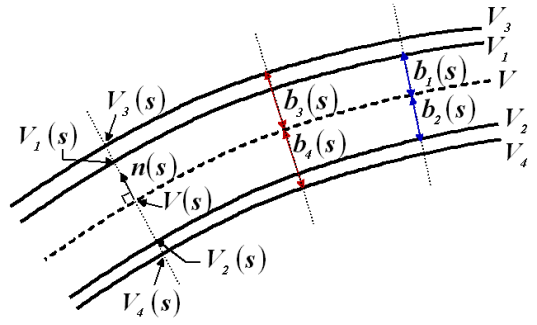

Figure 3: Parametric representation of the proposed model.

this line is considered to be fixed and will therefore no longer evolve in the subsequent steps. Additionally, we choose to model the walls as four curves approximately parallel to this line. We respectively denote by $V_{1}, V_{2}$ and $V_{3}, V_{4}$ the inner and outer borders of walls, and define them by

$$
\left\{\begin{array}{l}
V_{1}(s)=V(s)+b_{1}(s) \vec{n}(s) \\
V_{2}(s)=V(s)-b_{2}(s) \vec{n}(s) \\
V_{3}(s)=V(s)+b_{3}(s) \vec{n}(s) \\
V_{4}(s)=V(s)-b_{4}(s) \vec{n}(s),
\end{array}\right.
$$

where $\vec{n}(s)$ is the normal vector to the curve $V$ and $b_{k}(s)$ is the local distance between the reference line $V$ and the curve $V_{k}, \forall k \in\{1, \ldots, 4\}$ (see Figure 3). This model allows a direct correspondence between each curve $V_{k}$ and the reference line $V$. In what follows, we first detail how these curves are roughly positioned near the borders of walls. Next, we describe the model used to refine their positioning.

\section{A. Pre-segmentation}

First, we discretize the reference line $V$ in $m$ equally spaced points and denote by $V_{i}=(x(s=i h), y(s=i h))^{T}$ the discrete coordinates at the point $V_{i}$ and $\vec{n}_{i}$ the associated normal vector ( $h$ is the discretization step). We also discretize the curves representing the walls with

$$
\left\{\begin{aligned}
V_{i}^{1}\left(b_{i}^{\text {int }}\right) & =V_{i}+b_{i}^{\text {int }} \vec{n}_{i} \\
V_{i}^{2}\left(b_{i}^{\text {int }}\right) & =V_{i}-b_{i}^{i n t} \vec{n}_{i} \\
V_{i}^{3}\left(b_{i}^{\text {ext }}\right) & =V_{i}+b_{i}^{\text {ext }} \vec{n}_{i} \\
V_{i}^{4}\left(b_{i}^{\text {ext }}\right) & =V_{i}-b_{i}^{\text {ext }} \vec{n}_{i}
\end{aligned}\right.
$$

where $b^{i n t}$ and $b^{\text {ext }}$ respectively denote the half-diameter of the inner and outer borders. The model (3) assumes that the inner and outer borders lie at the same distance from the reference line $V$. Although this assumption could appear to be strong, it is verified for a large number of the images presented in Section I. Also, the presegmentation described below leads to a preliminary result which will be refined in Section III-B.

Let us now denote by $I$ a grayscale image with values in $[0,1]$. We denote by $D_{\vec{u}} I(p)$ the derivative of $I$ in the direction $\vec{u}$ at the point $p$. Moreover, for an half-diameter $b \in \mathbb{R}^{+}$, a point $V_{i}$ and a window of size $(2 r+1)$, we define the mean intensity along the curves $V^{1}$ and $V^{2}$ by

$$
\bar{I}^{i n t}(b, i, r)=\frac{1}{2(2 r+1)} \sum_{j=-r}^{+r}\left(I\left(V_{i+j}^{1}(b)\right)+I\left(V_{i+j}^{2}(b)\right)\right),
$$

the mean local gradient along the curves $V^{1}$ and $V^{2}$ by

$$
\begin{array}{r}
\bar{D}^{i n t}(b, i, r)=\frac{1}{2(2 r+1)} \sum_{j=-r}^{+r}\left(D_{\vec{n}_{i+j}} I\left(V_{i+j}^{1}(b)\right)+\right. \\
\left.D_{-\vec{n}_{i+j}} I\left(V_{i+j}^{2}(b)\right)\right),
\end{array}
$$

and the mean local gradient along the curves $V^{3}$ and $V^{4}$ by

$$
\begin{array}{r}
\bar{D}^{e x t}(b, i, r)=\frac{1}{2(2 r+1)} \sum_{j=-r}^{+r}\left(\left|D_{\vec{n}_{i+j}} I\left(V_{i+j}^{3}(b)\right)\right|+\right. \\
\left.\left|D_{-\vec{n}_{i+j}} I\left(V_{i+j}^{4}(b)\right)\right|\right) .
\end{array}
$$

Increasing the window radius $r$ makes gradient measures more robust to noise but less reliable where strong deformations occur along vessels. This parameter therefore requires a tradeoff. Due to the profile of lumens and since we do not use absolute values in (5), we expect this quantity to be larger near the inner borders of walls than near the axial reflection. We now summarize the steps necessary to estimate the halfdiameters $b^{\text {int }}$ and $b^{\text {ext }}$ in (3).

Step 1 


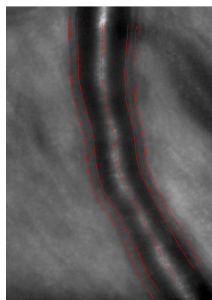

(a)

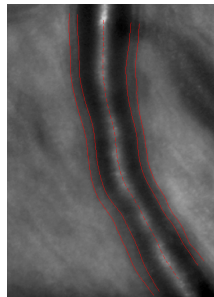

(b)

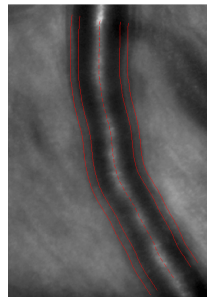

(c)

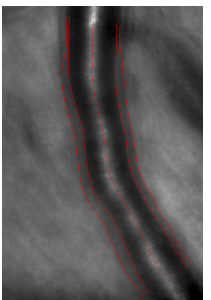

(d)
Figure 4: Benefit of using a presegmentation with varying halfdiameters (a),(b) against constant ones (c),(d) for a pathological case. Here, we set $r=10$ and $\alpha=0.95$. In (a) and (c), the image is superimposed with the presegmentations while it is superimposed in (b) and (d) with the segmentations obtained using the model described in Section III-B.

We jointly search for a constant half-diameter of inner and outer borders maximizing the mean gradient divided by the mean intensity along the curves $V^{1}, V^{2}, V^{3}$ and $V^{4}$. Using Equations (4), (5) and (6), such half-diameters are given by

$$
\begin{aligned}
\left(b^{\text {int }}, b^{\prime e x t^{*}}\right)=\underset{\substack{b^{\prime i n t}, b^{\prime} \text { ext } \\
b^{\prime i n t}<b^{\prime e x t}}}{\operatorname{argmax}}\left[\frac{\sum_{i=1}^{m} \bar{D}^{i n t}\left(b^{\text {int }}, i, r\right)}{\sum_{i=1}^{m} \bar{I}^{i n t}\left(b^{\prime i n t}, i, r\right)}+\right. \\
\left.\frac{1}{m} \sum_{i=1}^{m} \bar{D}^{\text {ext }}\left(b^{\text {ext }}, i, r\right)\right],
\end{aligned}
$$

where we remind that $m$ is the number of discrete points on $V$. Dividing by the intensity encourages gradients in dark areas. To speed up this step, the search interval for $b^{\prime e x t}$ is restricted to typical Wall-to-Lumen Ratio values [10]. Such a simplistic approach can however fail to accurately segment the walls when deformations occur along vessels. Estimating varying half-diameters for inner and outer borders is therefore crucial to take into account these deformations (see Figure 4).

\section{Step 2}

We search for the position along the vessels having the largest contrast along the curves defined by the half-diameters $b^{\prime i n t^{*}}$ and $b^{\prime e x t^{*}}$ found at Step 1 . This position is given by

$$
i^{*}=\underset{i}{\operatorname{argmax}} \frac{1}{2}\left(\bar{D}^{i n t}\left(b^{\prime i n t^{*}}, i, r\right)+\bar{D}^{\text {ext }}\left(b^{\prime e x t^{*}}, i, r\right)\right) \text {. }
$$

\section{Step 3}

Finally, we jointly search for a variable half-diameter of inner and outer borders whose difference (the wall thickness) is constant and having the largest contrast along the curves defined by the half-diameters $b^{\prime i n t^{*}}$ and $b^{\prime e x t^{*}}$ found at Step 1 . These half-diameters are constructed iteratively under a regularity constraint from each side of the position $i^{*}$ found at Step 2. For a fixed gap $e$, the half-diameter of inner borders $b^{\prime \prime i n t^{*}}$ is constructed as follows:

$$
b_{i}^{\prime \prime i n t^{*}}= \begin{cases}b^{\prime i n t^{*}} & \text { if } i=i^{*} \\ \operatorname{argmax}_{b_{i}^{\prime \prime i n t}} E\left(b_{i}^{\prime \prime i n t}, b_{i-1}^{\prime \prime i n t^{*}}, i, e, r\right) & \text { if } i>i^{*} \\ \operatorname{argmax}_{b_{i}^{\prime \prime \text { int }}} E\left(b_{i}^{\prime \prime i n t}, b_{i+1}^{\prime \prime i n t^{*}}, i, e, r\right) & \text { if } i<i^{*},\end{cases}
$$

with

$$
\begin{aligned}
& E\left(b_{i}^{\prime \prime i n t}, b_{j}^{\prime \prime i n t^{*}}, i, e, r\right)=\alpha\left(\frac{\bar{D}^{i n t}\left(b_{i}^{\prime \prime i n t}, i, r\right)}{\bar{I}^{\text {int }}\left(b_{i}^{\prime \prime i n t}, i, r\right)}\right. \\
& \left.+\bar{D}^{e x t}\left(b_{i}^{\prime \prime i n t}+\bar{e}+e, i, r\right)\right)+(1-\alpha)\left(b_{i}^{\prime \prime i n t}-b_{j}^{\prime \prime i n t^{*}}\right)^{2}
\end{aligned}
$$

where $\bar{e}=\left(b^{\prime e x t^{*}}-b^{\prime i n t^{*}}\right)$ and $\alpha \in[0,1]$ a parameter controlling the amount of regularity. In (9), notice that the term on the left has the same form as (7), except that it only concerns a single point. Finally, we search the best gap with

$$
e^{*}=\underset{e}{\operatorname{argmax}} \sum_{i>0} E\left(b_{i}^{\prime \prime i n t^{*}}, b_{i-1}^{\prime \prime i n t^{*}}, i, e, r\right),
$$

and where $b^{\prime \prime i n t^{*}}$ is constructed using (8) and (9). Notice that the discretization step of the search intervals of (8) and (10) is divided by two. The half-diameters estimates of inner and outer borders are finally respectively given by $b^{\text {int }}=b^{\prime \prime i n t^{*}}$ and $b^{\text {ext }}=b^{\prime \prime i n t^{*}}+\left(b^{\prime e x t^{*}}-b^{\prime i n t^{*}}\right)+e^{*}$. Rough estimates of inner and outer borders are now fully defined using (3).

\section{B. Refined segmentation}

The model proposed in [11] simultaneously evolves two curves under a parallelism constraint. In what follows, we describe an extension of this model for extracting four curves $V_{1}, V_{2}, V_{3}$ and $V_{4}$ almost parallel to a reference line $V$. Since this line is fixed in our situation, the energy becomes

$$
E\left(V_{1}, \ldots, V_{4}, b_{1}, \ldots, b_{4}\right)=\sum_{k=1}^{4}\left(E_{\text {Image }}\left(V_{k}\right)+R\left(V_{k}, b_{k}\right)\right)
$$

where the term

$$
E_{\text {Image }}\left(V_{k}\right)=\int_{0}^{1} P\left(V_{k}(s)\right) d s
$$

is designed to attract the curve $V_{k}$ towards large intensity gradients (see [16]). In this context, the term $E_{\text {Image }}$ is based on the Gradient Vector Flow [17]. The role of the term $R$ in (11) is to control the variation of the distance $b_{k}$, thus imposing a local parallelism. The authors of [11] proposed a function of the derivative of $b_{k}$ with

$$
R\left(V_{k}, b_{k}\right)=\int_{0}^{1} Q\left(s, b_{k}^{\prime}\right) d s=\int_{0}^{1} \varphi_{k}(s)\left(b_{k}^{\prime}(s)\right)^{2} d s,
$$

where $\varphi_{k}(s) \in \mathbb{R}^{+}$are application-dependent parameters that locally control the strength of the parallelism of the curve $V_{k}$ with respect to the reference line $V$. More precisely, the larger the parameter $\varphi_{k}(s)$ is, the more strict is the parallelism to the reference line $V$. It is worth underlying that the distance between both curves has not to be known. It is adjusted during the evolution process and can vary along boundaries. Notice also that the energy (11) does not ensure that $b_{1}(s)<b_{3}(s)$ and $b_{2}(s)<b_{4}(s)$. However, we never encountered such a behavior in our experiments (and in particular, for those detailed in Section IV).

Since the energy (11) does not have crossing terms involving different curves, the minimization can be independently 
done for each curve $V_{k}$. For any $k \in\{1, \ldots, 4\}$, the EulerLagrange equation expresses the minimization of (11) with respect to $b_{k}(s)$

$$
\frac{\partial P\left(V_{k}(s)\right)}{\partial b_{k}}-\frac{d}{d s} \frac{\partial Q\left(s, b_{k}^{\prime}\right)}{\partial b_{k}^{\prime}}=0,
$$

and the evolution of the distance $b_{k}$ to the line $V$ is driven by

$$
\left\langle\vec{n},-\nabla P\left(V_{k}(s)\right)\right\rangle-2\left[\varphi_{k}(s) b_{k}^{\prime \prime}(s)+\varphi_{k}^{\prime}(s) b_{k}^{\prime}(s)\right]=0 .
$$

The latter equation is solved by discretizing it and introducing the time variable using standard numerical approximations of derivatives (central difference in space, backward difference in time). The resolution of the above equations stops when

$$
\max _{k \in\{1, \ldots, 4\}}\left\{\max _{s}\left|b_{k}^{n}(s)-b_{k}^{n-1}(s)\right|\right\} \leq \varepsilon .
$$

In the latter expression, $b_{k}^{n}(s)$ is the estimate of the distance of the curve $V_{k}$ to the reference line $V$ at iteration $n$ and $\varepsilon \simeq 0$ is an accuracy parameter. An example of resolution using this model is illustrated in Figure 4(c).

\section{EVALUATION}

Thirteen images from healthy subjects were manually delineated by three physicians. These physicians have several years of experience in the field of AO image interpretation. The images were selected to ensure the representativeness of the quality and the noise levels encountered by physicians during routine clinical. Let us denote resp. by $V^{M}$ and $V^{A}$ a manual segmentation and an automatic segmentation. For each image, using the same axial reflection, we measure the absolute relative difference on the inner diameter, the outer diameter and the total wall thickness (i.e. the difference between outer and inner diameters), resp. defined for each point by

$$
\begin{gathered}
\delta_{i n t}\left(V^{M}, V^{A}\right)=\frac{\left|d_{i n t}\left(V^{M}\right)-d_{i n t}\left(V^{A}\right)\right|}{d_{i n t}\left(V^{M}\right)} \times 100 \\
\delta_{e x t}\left(V^{M}, V^{A}\right)=\frac{\left|d_{e x t}\left(V^{M}\right)-d_{e x t}\left(V^{A}\right)\right|}{d_{e x t}\left(V^{M}\right)} \times 100 \\
\delta_{w t}\left(V^{M}, V^{A}\right)=\frac{\left|d_{w t}\left(V^{M}\right)-d_{w t}\left(V^{A}\right)\right|}{d_{w t}\left(V^{M}\right)} \times 100
\end{gathered}
$$

where $d_{i n t}, d_{\text {ext }}$ and $d_{w t}$ denote resp. the inner diameter, the outer diameter and the total wall thickness. The measure $\delta_{w t}$ is of great importance for us due to its high sensitivity. Also, measurements were only taken into account where no vessel bifurcation occurs (see Figure 5). For the parameters, we use $\varepsilon=0.1, \alpha=0.9, r=10$ and $\varphi_{k}=100$, $\forall k \in\{1, \ldots, 4\}$. Also, we choose manual segmentations from the most experienced physician as a reference (denoted by Phys $R$ f $)$. For each image, we then computed the mean and standard deviation of (13), (14) and (15) between the segmentations obtained by the automatic procedure and the those from the physician Phys ${ }_{\text {Ref }}$. The same statistics were also computed between the segmentations from physicians (different of Phys $s_{\text {Ref }}$ ) and those from the physician Phys Ref. The results of these experiments are detailed in Table I and illustrated in Figure 5. To put in perspective these results, we provide between parentheses in Table I, the above statistics but for a displacement of one pixel all along a curve. In that case, the numerators of (13), (14) and (15) become equal to one.
Due to the size of arterial walls, we first remark that the error (i.e. mean and standard deviation) is much larger on the total wall thickness compared to inner and outer diameters. Noticeably, the error introduced by the automatic procedure is globally smaller than the inter-physicians error on total wall thickness and outer diameter. Although this is not the case concerning the inner diameter, the errors remain globally very near it. For inner / outer diameters and total wall thickness measurements between the automatic procedure and the physician Phys $s_{\text {Ref }}$, the standard deviation is globally smaller than the mean. This means a good robustness of the proposed approach. For some subjects, the error introduced by the automatic procedure is larger than the inter-physicians error. For some of them, we do believe that this is due to a lack of accuracy in manual segmentations. Due to the poor contrast along walls and the size of these structures, it is indeed difficult for physicians to delineate them all along a vessel with high accuracy (see Subject 5). For other subjects, the outer border of walls computed by the automatic procedure slightly differs from manual segmentations. This is for instance the case for the Subject 3 where a kind of double contour occurs all along the vessel. However, there is currently no consensus among physicians about the ideal location of the outer border of walls in such situation. Finally, we are currently evaluating the proposed approach on pathological subjects. First experiments show a good robustness with respect to morphological deformations.

\section{REFERENCES}

[1] N.H. Buus et al. Small artery structure during antihypertensive therapy is an independent predictor of cardiovascular events in essential hypertension. Journal of Hypertension, 31(4):791-797, 2013.

[2] A.M. Heagerty et al. Small artery structure in hypertension: Dual processes of remodeling and growth. Journal of Hypertension, 21:391397, 1993.

[3] H. Taylor and J. Keeffe. World blindness: A 21st century perspective. British Journal of Ophtalmology, 85(3):261-266, 2001.

[4] F. Rossant, M. Badellino, A. Chavillon, I. Bloch, and M. Paques. A morphological approach for vessel segmentation in eye fundus images, with quantitative evaluation. Journal of Medical Imaging and Health Informatics, 1(1):42-49, 2011.

[5] C. Kulcsár, G. Le Besnerais, E. Ödlund, and X. Levecq. Robust processing of images sequences produced by an adaptive optics retinal camera. In Optical Society of America, Adaptive Optics: Methods, Analysis and Applications, page OW3A.3, 2013.

[6] C. Viard, K. Nakashima, B. Lamory, X. Paques, M. ad Levecq, and N. Château. Imaging microscopic structures in pathological retinas using a flood-illumination adaptive optics retinal camera. In Photonics West: Biomedical Optics (BiOS), volume 7885, pages 788 509+, 2011.

[7] J. Liang, D.R. Williams, and D.T. Miller. Supernormal vision and highresolution retinal imaging through adaptive optics. Journal of Optical Society America A, 14(11):2884-2892, 1997.

[8] J.A. Martin and A. Roorda. Direct and noninvasive assessment of parafoveal capillary leukocyte velocity. Ophthalmology, 112(12):22192224, 2005.

[9] T.Y. Chan, D.A. Vannasdale, and S.A. Burns. The use of forward scatter to improve retinal vascular imaging with an adaptive optics scanning laser ophthalmoscope. Biomedical Optics Express, 3(10):2537-2549, 2012

[10] E. Koch. Morphometric study of human retina arteriolars in high resolution imaging. Master's thesis, Université Pierre et Marie Curie, 2012.

[11] I. Ghorbel, F. Rossant, I. Bloch, and M. Paques. Modeling a parallelism constraint in active contours. Application to the segmentation of eye vessels and retinal layers. In International Conference on Image Processing (ICIP), pages 445-448, 2011. 

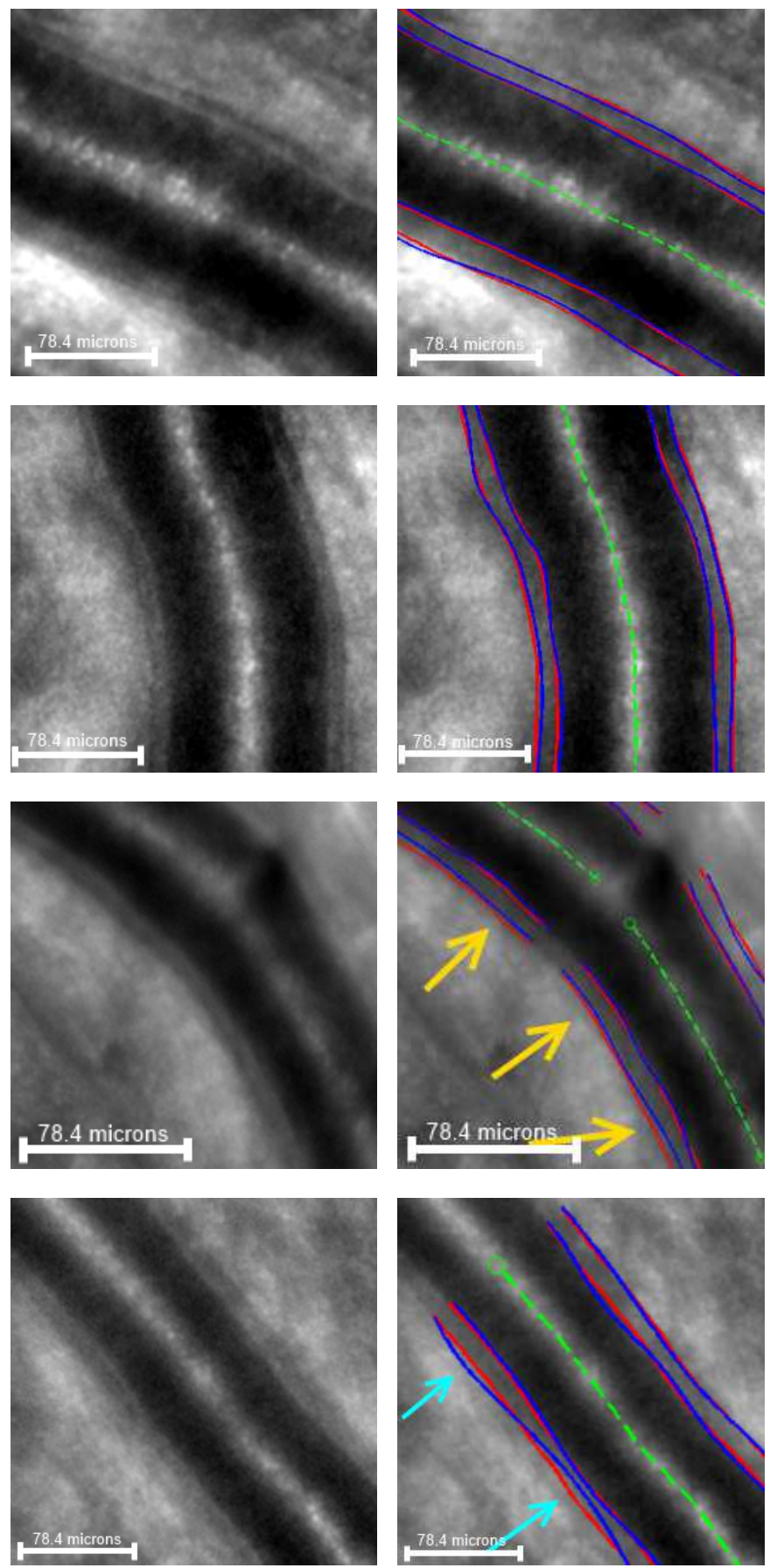

Figure 5: From top to bottom: positive (half-up) and negative (half-down) results against the reference physician Phys ${ }_{\text {Ref }}$ for Subjects 7, 11, 3 and 5 (see Table I). The manual and the automatic segmentations are drawn in blue and red, respectively. The green dashed line is the reference line. The original images are provided in the left column while they are superimposed to the segmentations in the right column. Yellow and cyan arrows point misplacement of borders in automatic and manual segmentations, respectively.

\begin{tabular}{|c|c|c|}
\hline Subject & Inter-physicians & Our method / Phys $R e f$ \\
\hline 1 & $2.29 \pm 1.54(1.13 \pm 0.04)$ & $2.07 \pm 1.06(1.13 \pm 0.04)$ \\
\hline 2 & $3.50 \pm 2.37(2.47 \pm 0.16)$ & $4.17 \pm 3.29(2.47 \pm 0.16)$ \\
\hline 3 & $2.86 \pm 2.57(1.37 \pm 0.04)$ & $1.63 \pm 1.31(1.37 \pm 0.04)$ \\
\hline 4 & $2.29 \pm 1.7(0.75 \pm 0.03)$ & $3.16 \pm 1.65(0.75 \pm 0.03)$ \\
\hline 5 & $2.79 \pm 1.91(0.89 \pm 0.05)$ & $3.21 \pm 2.09(0.89 \pm 0.05)$ \\
\hline 6 & $3.75 \pm 2.8(1.13 \pm 0.05)$ & $2.14 \pm 2.34(1.13 \pm 0.05)$ \\
\hline 7 & $2.82 \pm 2.38(0.87 \pm 0.04)$ & $2.51 \pm 2.64(0.87 \pm 0.04)$ \\
\hline 8 & $2.84 \pm 2.41(0.97 \pm 0.03)$ & $3.78 \pm 2.92(0.97 \pm 0.03)$ \\
\hline 9 & $3.26 \pm 2.65(0.87 \pm 0.03)$ & $4.58 \pm 3.19(0.87 \pm 0.03)$ \\
\hline 10 & $3 \pm 2.08(0.8 \pm 0.02)$ & $1.87 \pm 1.96(0.8 \pm 0.02)$ \\
\hline 11 & $2.75 \pm 1.8(0.84 \pm 0.02)$ & $2.36 \pm 1.88(0.84 \pm 0.02)$ \\
\hline 12 & $2.7 \pm 2.26(0.9 \pm 0.04)$ & $2.51 \pm 2.05(0.9 \pm 0.04)$ \\
\hline 13 & $3.38 \pm 2.27(0.84 \pm 0.05)$ & $6.07 \pm 4.25(0.84 \pm 0.05)$ \\
\hline \hline Overall & $2.92 \pm 2.24(0.99 \pm 0.35)$ & $3.06 \pm 2.73(0.99 \pm 0.35)$ \\
\hline
\end{tabular}

\begin{tabular}{|c|c|c|}
\hline Subject & Inter-physicians & Our method / Phys ${ }_{\text {Ref }}$ \\
\hline 1 & $2.08 \pm 1.69(0.91 \pm 0.02)$ & $2.06 \pm 1.83(0.91 \pm 0.02)$ \\
\hline 2 & $2.2 \pm 1.76(1.7 \pm 0.07)$ & $3.86 \pm 1.92(1.7 \pm 0.07)$ \\
\hline 3 & $2.16 \pm 1.53(1.05 \pm 0.03)$ & $5.64 \pm 2.82(1.05 \pm 0.03)$ \\
\hline 4 & $2.8 \pm 1.94(0.6 \pm 0.02)$ & $1.57 \pm 1.04(0.6 \pm 0.02)$ \\
\hline 5 & $2.94 \pm 2.19(0.67 \pm 0.03)$ & $2.28 \pm 1.66(0.67 \pm 0.03)$ \\
\hline 6 & $5.11 \pm 3.81(0.74 \pm 0.03)$ & $3.05 \pm 2.1(0.74 \pm 0.03)$ \\
\hline 7 & $4.05 \pm 2.59(0.65 \pm 0.01)$ & $1.96 \pm 1.38(0.65 \pm 0.01)$ \\
\hline 8 & $1.93 \pm 1.36(0.69 \pm 0.02)$ & $2.23 \pm 1.34(0.69 \pm 0.02)$ \\
\hline 9 & $2.93 \pm 1.84(0.62 \pm 0.01)$ & $2.33 \pm 1.01(0.62 \pm 0.01)$ \\
\hline 10 & $3.03 \pm 1.97(0.6 \pm 0.01)$ & $1.67 \pm 1.45(0.6 \pm 0.01)$ \\
\hline 11 & $2.18 \pm 1.95(0.68 \pm 0.01)$ & $2.19 \pm 1.33(0.68 \pm 0.01)$ \\
\hline 12 & $2.48 \pm 1.54(0.69 \pm 0.03)$ & $5.04 \pm 2.27(0.69 \pm 0.03)$ \\
\hline 13 & $1.7 \pm 1.28(0.68 \pm 0.02)$ & $2.95 \pm 2.68(0.68 \pm 0.02)$ \\
\hline \hline Overall & $2.83 \pm 2.31(0.74 \pm 0.24)$ & $2.7 \pm 2.16(0.74 \pm 0.24)$ \\
\hline
\end{tabular}

\begin{tabular}{|c|c|c|}
\hline Subject & Inter-physicians & Our method / Phys ${ }_{\text {Ref }}$ \\
\hline 1 & $10.57 \pm 8.1(4.68 \pm 0.59)$ & $9.12 \pm 6.33(4.68 \pm 0.59)$ \\
\hline 2 & $8.78 \pm 6.59(5.53 \pm 0.58)$ & $9.79 \pm 6.03(5.53 \pm 0.58)$ \\
\hline 3 & $11.63 \pm 9.54(4.63 \pm 0.45)$ & $23.11 \pm 13.1(4.63 \pm 0.45)$ \\
\hline 4 & $14.52 \pm 11.95(3.04 \pm 0.43)$ & $13.52 \pm 8.35(3.04 \pm 0.43)$ \\
\hline 5 & $17.46 \pm 14.5(2.8 \pm 0.57)$ & $15.62 \pm 11(2.8 \pm 0.57)$ \\
\hline 6 & $14.32 \pm 10.93(2.2 \pm 0.29)$ & $10.9 \pm 7.87(2.2 \pm 0.29)$ \\
\hline 7 & $16.12 \pm 10.57(2.54 \pm 0.33)$ & $9.82 \pm 8.59(2.54 \pm 0.33)$ \\
\hline 8 & $9.85 \pm 7.64(2.46 \pm 0.22)$ & $12.22 \pm 6.22(2.46 \pm 0.22)$ \\
\hline 19 & $14.77 \pm 12.36(2.17 \pm 0.32)$ & $11.42 \pm 5.6(2.17 \pm 0.32)$ \\
\hline 10 & $13.17 \pm 8.52(2.36 \pm 0.23)$ & $7.4 \pm 5.87(2.36 \pm 0.23)$ \\
\hline 11 & $15.98 \pm 13.26(3.67 \pm 0.36)$ & $15.15 \pm 13.8(3.67 \pm 0.36)$ \\
\hline 12 & $12.58 \pm 10.11(2.88 \pm 0.22)$ & $25.52 \pm 8.41(2.88 \pm 0.22)$ \\
\hline 13 & $15.69 \pm 10.53(3.5 \pm 0.4)$ & $14.68 \pm 11.8(3.49 \pm 0.4)$ \\
\hline \hline Overall & $14.09 \pm 11.34(3.1 \pm 0.98)$ & $13.84 \pm 10.52(3.1 \pm 0.98)$ \\
\hline
\end{tabular}

Table I: From top to bottom: inter-physicians and automatic / Phys ${ }_{\text {Ref }}$ absolute relative error for inner diameter outer diameter and total wall thickness. The numbers between parentheses denote a unit displacement all along the curve (see text).

[12] E. Koch, D. Rosenbaum, A. Brolly, J.-A. Sahel, P. Chaumet-Riffaud, X. Girard, F. Rossant, and M. Paques. Morphometric analysis of small arteries in the human retina using adaptive optics imaging: Relationship with blood pressure and focal vascular changes. Journal of Hypertension, 2013.

[13] J. Weickert, B.H. Romeny, and M. Viergever. Efficient and reliable schemes for nonlinear diffusion filtering. IEEE Transactions on Image Processing, 7(3):398-410, 1998.

[14] L.D. Cohen and R. Kimmel. Global minimum for active contour models: A minimal path approach. International Journal of Computer Vision, 24(1):57-78, 1997.

[15] J.A. Sethian. Level Set Methods and Fast Marching Methods: Evolving Interfaces in Computational Geometry, Fluid Mechanics, Computer Vision, and Materials Science. Cambridge University Press, 1999.

[16] M. Kass, A. Witkin, and D. Terzopoulos. Snakes: Active contour models. International Journal of Computer Vision, 1(4):321-331, 1988.

[17] C. Xu and J.L. Prince. Snakes, shapes and gradient vector flow. IEEE Transactions on Image Processing, 7(3):359-369, 1998. 